\title{
Herpesvirus bovino 1 (BoHV-1): actualización de las cepas circulantes en Argentina
}

\author{
Maidana, S.S. ${ }^{1,2,3}$; Marin, M. ${ }^{2,5}$; Destefano, G. ${ }^{1}$; Combessies, G. ${ }^{6}$; Romera, S.A. ${ }^{1,2,3,4}$ \\ ${ }^{1}$ Instituto de Virología INTA Hurlingham, Buenos Aires, Argentina. \\ ${ }^{2}$ CONICET. ${ }^{3}$ Cátedra Inmunogenética Univ. Morón. ${ }^{4}$ Cát. Inmunología \\ Univ.Salvador, Pilar. ${ }^{5}$ INTA Balcarce, ${ }^{6}$ Lab.Azul, Buenos Aires, Argentina. \\ E-mail: maidana.silvina@inta.gob.ar
}

\begin{abstract}
Resumen
Maidana, S.S.; Marin, M.; Destefano, G.; Combessies, G.; Romera, S.A.: Herpesvirus bovino 1 (BoHV-1): actualización de las cepas circulantes en Argentina. Rev. vet. 29: 1, 5256, 2018. BoHV-1 genera en el ganado bovino una amplia gama de manifestaciones clínicas, tales como la rinotraqueítis infecciosa bovina, abortos y vulvovaginitis pustular infecciosa, que dependen de la virulencia del virus y del estado inmunitario del hospedador. En este trabajo se caracterizaron en Argentina 44 aislamientos de BoHV-1 por el análisis de restricción con endonucleasas (REA). El ADN viral fue sometido al clivaje de la enzima HindIII, la cual permite la diferenciación entre los subtipos 1.1, 1.2a y 1.2b. De acuerdo al perfil REA de los aislamientos, 35 cepas $(80 \%)$ mostraron un patrón similar a la cepa K22 (prototipo BoHV$1.2 b), 7$ cepas (16\%) exhibieron un patrón similar a la cepa LA (prototipo BoHV-1.1) y sólo 2 cepas (4\%) revelaron un patrón similar a la cepa ST (prototipo BoHV-1.2a). Los resultados demuestran una mayor prevalencia del subtipo $1.2 \mathrm{~b}$ entre los aislamientos BoHV-1 argentinos. Además, se reporta por primera vez en Argentina, la circulación del subtipo BoHV-1.2a.
\end{abstract}

Palabras clave: bovino, herpesvirus, subtipos, análisis del perfil de restricción por endonucleasas.

\begin{abstract}
Maidana, S.S.; Marin, M.; Destefano, G.; Combessies, G.; Romera, S.A.: Bovine herpesvirus 1 (BoHV-1): update of circulating strains in Argentina. Rev. vet. 29: 1, 52-56, 2018. BoHV-1 in cattle results in a wide range of clinical manifestations, such as infectious bovine rhinotracheitis, abortion and infectious pustular vulvovaginitis, depending on the virulence of the virus and the immune status of the host. Forty-four Argentinean BoHV1 isolates were characterized by restriction endonuclease analysis (REA). Viral DNA was cleaved with HindIII which allows the differentiation among subtypes 1.1, 1.2a and 1.2b. According to the REA, 35 field strains $(80 \%)$ showed a pattern similar to K22 strain (BoHV-1.2b prototype), 7 field strains (16\%) showed a clear pattern similar to LA strain (BoHV-1.1 prototype) and only 2 filed strains (4\%) showed a clear pattern similar to ST strain (BoHV-1.2a prototype). The results demonstrated the presence of a marked prevalence of subtype $1.2 \mathrm{~b}$ among Argentinean BoHV-1 isolates. We also report for the first time in Argentina the circulation of the subtype BoHV-1.2a.
\end{abstract}

Key words: bovine, herpesvirus, subtypes, restriction endonuclease analysis.

\section{INTRODUCCIÓN}

El BoHV-1, miembro de la familia Herpesviridae, afecta naturalmente a la especie bovina, provocando una amplia gama de manifestaciones: rinotraqueítis (IBR), vulvovaginitis pustular infecciosa (IPV), balanopostitis (IPB), conjuntivitis, aborto, enteritis y encefalitis ${ }^{7}$.

BoHV-1 es un patógeno mundialmente diseminado que muestra diferencias significativas en la incidencia

Recibido: 12 octubre 2017 / Aceptado: 7 marzo 2018 regional y la prevalencia respecto de las posiciones geográficas y el manejo de cría bovina en cada establecimiento ${ }^{7}$.

Así como otras enfermedades causadas por herpesvirus en el hombre y en los animales, el ciclo de reactivación de la latencia tiene un profundo impacto epidemiológico ya que es responsable del mantenimiento de BoHV-1 en la población bovina ${ }^{12}$.

Todas las cepas de BoHV-1 se clasifican en tres subtipos BoHV-1.1, BoHV-1.2a y BoHV-1.2b ${ }^{7}$. Aunque la mayoría de las cepas de BoHV-1.1 han sido aisladas de enfermedades respiratorias y las cepas de subtipo 
BoHV-1.2 a partir de lesiones de órganos genitales, el único criterio distintivo fiable es el análisis del perfil de restricción del ADN viral por endonucleasa ${ }^{6}$.

Históricamente los subtipos 1.1 y $1.2 \mathrm{a}$ se han asociado con enfermedades graves, incluida la infección del feto y el aborto ${ }^{6}$. Sin embargo el subtipo $1.2 \mathrm{~b}$ generalmente no se ha asociado con el aborto ${ }^{3}$. De hecho, los terneros infectados experimentalmente por vía nasal con cepas de BoHV-1.2 mostraron signos clínicos respiratorios y fueron capaces de transmitir la infección respiratoria a otro terneros ${ }^{3}$. Del mismo modo, las lesiones del tracto reproductivo en novillas se observaron después de la inoculación intrauterina con BoHV-1.1 ${ }^{6}$.

En cuanto al impacto económico, el BoHV-1 es responsable de pérdidas significativas ocasionadas por las enfermedades y las restricciones comerciales de la industria ganadera ${ }^{7}$. Por lo tanto, los programas de control se desarrollaron rápidamente después de la aparición de IBR en rebaños de América del Norte, que generalmente se basan en el uso de vacunas marcadoras, en la detección y el sacrificio de animales seropositivos, o en la vacunación repetida de rebaños infectados.

Debido a la incapacidad de las vacunas para prevenir la infección por BoHV-1 y el establecimiento de latencia, los programas de control de BoHV-1 pueden durar un largo período antes de completar la erradicación de esta infección bien adaptada al ganado bovino ${ }^{7}$. Otra preocupación en los esquemas de erradicación de BoHV-1 es la capacidad de este virus para cruzar la barrera de especies huésped.

Los datos de campo y las infecciones experimentales han puesto de manifiesto la posible infección de varias especies de rumiantes con BoHV-1, así como la infección de bovinos con otros alfa herpesvirus de rumiantes, sin posibilidad de distinguirlos a nivel serológico y complejizando aún más la aplicación de los programas de erradicación ${ }^{7}$.

El análisis del perfil de restricción por endonucleasa (REA) se ha utilizado ampliamente para diferenciar los aislamientos de BoHV-1 ${ }^{7}$. Además, resultó ser particularmente útil para la diferenciación entre diversos alfaherpesvirus de rumiantes antigénicamente relacionados con BoHV-1, ya que muestran diferentes perfiles de restricción del ADN viral ${ }^{12}$. Esta técnica permite la subtipificación de aislamientos que aparentemente pertenecen al mismo virus.

Tradicionalmente, la diferenciación entre los subtipos de BoHV-1 se basa en las características clínicoepidemiológicas de los brotes seguidos de REA de ADN viral después del aislamiento viral ${ }^{1}$. En nuestro país se describió el primer brote de BoHV en 1982, a partir del cual se aislaron tanto BoHV-1, como la cepa de referencia A663 (BoHV-5b) clasificada como BoHV$5^{11}$ y ahora reportada como virus recombinante natural entre BoHV-1/5 (Maidana S.S. et al.: World Buiatric Congress, Dublin, 2016).

Por lo tanto, los objetivos de este estudio fueron actualizar, caracterizar y comparar por REA las variantes genéticas de los aislamientos argentinos de BoHV-1 obtenidos a partir de bovinos con diferentes signos clínicos desde 1984 hasta 2015.

\section{MATERIAL Y MÉTODOS}

Virus y células. Las cepas de campo BoHV-1 se obtuvieron por aislamiento a partir de muestras de animales naturalmente infectados. Los detalles del sitio geográfico, el tiempo, el tipo de muestra de la que se obtuvo y la sintomatología del animal al momento de la toma de muestra se encuentran descriptos en la Tabla 1.

Las muestras fueron colectadas y aisladas previamente por nuestro grupo o bien se utilizaron aislamientos del banco de cepas del laboratorio de diagnóstico del Instituto de Virología del INTA Castelar; también se incluyeron aquéllas remitidos por distintos laboratorios privados de diagnóstico.

Se utilizaron células Madin Darby Bovine Kidney (MDBK) tanto para la amplificación de las cepas de referencia que se utilizaron como control, como para la detección de nuevos aislamientos. Las cepas virales de referencia utilizadas en los diversos ensayos fueron las siguientes: LA, K22, ST que corresponde a BoHV1.1, BoHV1.2b, BoHV1.2a respectivamente.

Obtención del ADN viral (PCR multiplex diferencial entre $B o H V-1 / 5$ y REA). Para la obtención de ADN viral, monocapas de células MDBK fueron crecidas en botellas de cultivo celular de $150 \mathrm{~cm}^{2}$ de superficie (T150) e infectadas con las cepas de referencia y los aislamientos de campo. Cuando el ECP fue extensivo, los frascos fueron congelados a $-70^{\circ} \mathrm{C}$.

Luego se realizó el clarificado a $3.000 \mathrm{rpm}$ durante 20 minutos a $4^{\circ} \mathrm{C}$. El sobrenadante fue ultracentrifugado a $100.000 \mathrm{~g}$ (Beckman L-90K) durante 1 hora a $4^{\circ} \mathrm{C}$. El pellet se resuspendió en $1 \mathrm{ml}$ de buffer TE (Tris 200 $\mathrm{mM}$ pH 8, EDTA $20 \mathrm{mM}$ ) con NP-40 0,1\% v/v (Sigma) e incubado a $37^{\circ} \mathrm{C}$ por 30 minutos y a $56^{\circ} \mathrm{C}$ por 1 hora. Luego se llevó a $30 \mathrm{ml}$ con TE, se agregaron $5 \mathrm{ml}$ de sacarosa $30 \%$ p/v en el fondo del tubo y se ultracentrifugó a $100.000 \mathrm{~g}$ durante 2 horas a $4^{\circ} \mathrm{C}$.

El pellet fue resuspendido en 500 ul de buffer de extracción (TE $\mathrm{pH}=8$, SDS $1 \%$, Proteinasa K 0,5 mg/ $\mathrm{ml})$. Para lograr la disolución del pellet, se incubó a $56^{\circ} \mathrm{C}$ por 2 horas. La purificación y extracción del ADN viral fueron realizadas según se detalla en un trabajo realizado en el norte de Francia ${ }^{12}$.

Primeramente se realizó una PCR multiplex disenada por M.Claus ${ }^{2}$, que diferencia entre BoHV-1/5. Los cebadores utilizados fueron $\mathrm{B} 1$ específico para BoHV-1 (5-CAA CCG AGA CGG AAA GCT CC-3_nt 185-204); B5 especifico para BoHV-5 (5-CGG ACG AGA CGC CCT TGG-3_nt 322-339) y un cebador consenso para ambos virus Bcon (5-AGT GCA CGT ACA GCG GCT CG-3_nt 519-538).

Los productos de amplificación, de diferentes pesos moleculares dependiendo del virus, corresponden al gen que codifica para glicoproteína gC (número de 
Tabla 1. Aislamientos de Bovine herpesvirus 1 argentinos y cepas de referencia agrupadas en subtipos de acuerdo con el análisis del perfil de restricción.

\begin{tabular}{|c|c|c|c|c|}
\hline subtipo & año & cepa & muestra & origen y signos clínicos \\
\hline \multirow[t]{34}{*}{$1.2 \mathrm{~b}$} & 1984 & $67 / 89$ & cerebro & Sin datos \\
\hline & 1989 & $107 / 89$ & hígado & Sin datos \\
\hline & \multirow[t]{2}{*}{1991} & LP 202 & hisopado prepucial & Sin datos \\
\hline & & LP 170 & hisopado prepucial & Sin datos \\
\hline & 1996 & $26 / 96$ & semen & Sin datos \\
\hline & 1998 & $98 / 759$ & hisop. nasal y ocular & Ayacucho, Buenos Aires. Sin datos. \\
\hline & 2001 & $01 / 156$ & secreción vaginal & Catriló, La Pampa. Aborto \\
\hline & 2001 & $01 / 211$ & secreción vaginal & Saladillo, Buenos Aires. Aborto \\
\hline & 2002 & $02 / 221$ & secreción vaginal & Balcarce, Buenos Aires. Aborto \\
\hline & 2002 & $02 / 701$ & hisopado prepucial & $\begin{array}{l}\text { Laprida, Buenos Aires. Presentación de toros con prepucio } \\
\text { inflamado y con lesiones en el glande peneano. }\end{array}$ \\
\hline & 2003 & $03 / 41$ & secreción vaginal & Ayacucho, Buenos Aires. Sin datos \\
\hline & 2003 & $03 / 134$ & $\begin{array}{l}\text { hisopado nasal y } \\
\text { ocular }\end{array}$ & $\begin{array}{l}\text { Balcarce, Buenos Aires. Lagrimeo, herpes labial, nariz seca, } \\
\text { diseños y pérdida de estatus, disfunción respirat. }\end{array}$ \\
\hline & 2004 & $16 / 04$ & semen & Entre Ríos. Asintomático \\
\hline & \multirow[t]{2}{*}{2006} & $67 / 06$ & semen & Saladillo, Buenos Aires. Asintomático \\
\hline & & $75 / 06$ & semen & San Antonio de Areco, Buenos Aires \\
\hline & 2009 & $09 / 210$ & hisopado nasal & Carlos Tejedor, Buenos Aires. Signos nerviosos \\
\hline & \multirow[t]{3}{*}{2010} & $621 / 10$ & semen & Saldungaray, Buenos Aires. Asintomático \\
\hline & & $612 / 10$ & semen & San Antonio de Areco, Buenos Aires. Asintomático \\
\hline & & $688 / 10$ & semen & San Antonio de Areco, Buenos Aires. Asintomático \\
\hline & \multirow[t]{3}{*}{2011} & 122232 & hisopado ocular & Queratoconjuntivitis \\
\hline & & $745 / 11$ & semen & Buenos Aires. Asintomático \\
\hline & & al-most $(890 / 11)$ & semen & Buenos Aires. Asintomático \\
\hline & \multirow[t]{8}{*}{2012} & 94 & hisopado ocular & Chubut, Esquel. Queratoconjuntivitis \\
\hline & & 146 & hisopado ocular & Chubut, Esquel. Queratoconjuntivitis \\
\hline & & 997 & hisopado ocular & Chubut, Esquel. Queratoconjuntivitis \\
\hline & & $932 / 12(50)$ & semen & Capitan Sarmiento, Buenos Aires. Asintomático \\
\hline & & $935 / 12(5)$ & semen & Capitan Sarmiento, Buenos Aires. Asintomático \\
\hline & & $935 / 12(17)$ & semen & Capitan Sarmiento, Buenos Aires. Asintomático \\
\hline & & $942 / 12(33)$ & semen & Capitan Sarmiento, Buenos Aires. Asintomático \\
\hline & & $972 / 12(30)$ & semen & Capitan Sarmiento, Buenos Aires. Asintomático \\
\hline & 2013 & $1039 / 13(36)$ & semen & Capitan Sarmiento, Buenos Aires. Asintomático \\
\hline & 2014 & $1127 / 14(58)$ & semen & Chaco. Asintomatico \\
\hline & \multirow[t]{2}{*}{2015} & $1273 / 15(61)$ & semen & San Antonio de Areco, Buenos Aires. Asintomático \\
\hline & & $1273 / 15(65)$ & semen & San Antonio de Areco, Buenos Aires. Asintomático \\
\hline \multirow[t]{2}{*}{$1.2 \mathrm{a}$} & 2012 & $972 / 12(26)$ & semen & San Antonio de Areco, Buenos Aires. Asintomático \\
\hline & 2015 & 39 & hisopado nasal & Mix herd (búfalo/cattle), Corrientes. Asintomático \\
\hline \multirow[t]{9}{*}{1.1} & 1984 & P 898 & cerebro y cornetes & Sin datos \\
\hline & 2003 & $03 / 404$ & secreción vaginal & Barker, Buenos Aires. Aborto \\
\hline & \multirow[t]{3}{*}{2007} & FR & cerebro & Encefalitis \\
\hline & & 40418 & cerebro & Encefalitis \\
\hline & & 41184 & cerebro & Encefalitis \\
\hline & 2008 & 86274 & hisopado nasal & Signos respiratorios \\
\hline & 2012 & $12 / 255$ & hisopado ocular & Lincoln, Buenos Aires. Conjuntivitis \\
\hline & \multirow[t]{2}{*}{2015} & $1260 / 15(67)$ & semen & San Antonio de Areco, Buenos Aires. Asintomático \\
\hline & & $1263 / 15(68)$ & semen & San Antonio de Areco, Buenos Aires. Asintomático \\
\hline
\end{tabular}

acceso al GenBank Z49223 and U35883). La PCR multiplex fue preparada en una mix de reacción $(12,5 \mathrm{ul}$ final) conteniendo $50 \mathrm{ng}$ de ADN viral, 0,4 pmol de cada cebador (B1, B5, and Bcon); 1,5 mM dNTPs (Invitrogen TM Life Technologies, USA); 2,5 units de Taq DNA Polimerasa (Invitrogen TM Life Technologies, USA); 1X PCR buffer (20 mM Tris- $\mathrm{HCl} \mathrm{pH} 8,4,50$
$\mathrm{mM} \mathrm{KCl}) ; 1,5 \mathrm{mM} \mathrm{MgCl} 2$ y agua ultrapura hasta llegar $12,5 \mu \mathrm{l}$.

La reacción de amplificación se realizó en un termo ciclador (Biometra TRIO - Thermoblock) bajo las siguientes condiciones: un paso de $10 \mathrm{~min}$ a $96^{\circ} \mathrm{C}$ seguido por 25 ciclos de $1 \mathrm{~min}$ a $96^{\circ} \mathrm{C} ; 1 \mathrm{~min}$ a $58^{\circ} \mathrm{C} ; 1 \mathrm{~min}$ a $72^{\circ} \mathrm{C}$ y un paso de extensión final de $10 \mathrm{~min}$ at $72^{\circ} \mathrm{C}$. 
Finalmente los productos de PCR fueron analizados en una corrida electroforética de agarosa $1 \%$, teñido con bromuro de etidio $(0,5 \mu \mathrm{g} / \mathrm{ml})$ en buffer TBE $\mathrm{pH} 8,4$ ( $89 \mathrm{mM}$ Tris, $89 \mathrm{mM}$ boric acid, 2 mM EDTA) y visualizado bajo luz UV.

Para el ensayo de REA, 4 ug de ADN viral fueron digeridos con la endonucleasa Hind-III siguiendo las recomendaciones del fabricante (Promega, Wisconsin, USA). Finalmente los productos de digestión fueron separados por electroforesis en gel de agarosa $0,7 \%$ a $50 \mathrm{~V}$ durante toda la noche usando buffer TBE. Luego el gel fue teñido con bromuro de etidio y el patrón de bandeo fue observado y fotografiado con el sistema de documentación de geles de BioRad.

\section{RESULTADOS}

En este estudio, analizamos un total de 44 cepas aisladas (entre 1984 y 2015) de bovinos de diferentes provincias. Los datos de las cepas se encuentran resumidos en la Tabla 1. Todas las cepas aisladas fueron exitosamente amplificadas en cultivo celular (MDBK). La PCR multiplex permitió diferenciar los aislamientos por peso molecular de los productos, $159 \mathrm{pb}$ para BoHV-5 y de 354 pb para BoHV-1.

A partir de las muestras analizadas se observó que la mayoría de los aislamientos de BoHV-1 fueron aislados a partir de semen (22 de 44) durante diagnósticos de rutina. La mayor parte de los aislamientos mostró un patrón de bandas por REA similar al prototipo BoHV-1.2b (35 aislamientos), 7 aislamientos fueron similares a BoHV-1.1 y los 2 últimos mostraron un patrón de bandas similar a BoHV-1.2a (Figura 1).

\section{DISCUSIÓN}

En América Latina la seroprevalencia de BoHV-1 es muy alta, con niveles que oscilan entre $20-90 \%$ dependiendo del país. Se ha informado que más del 75\%

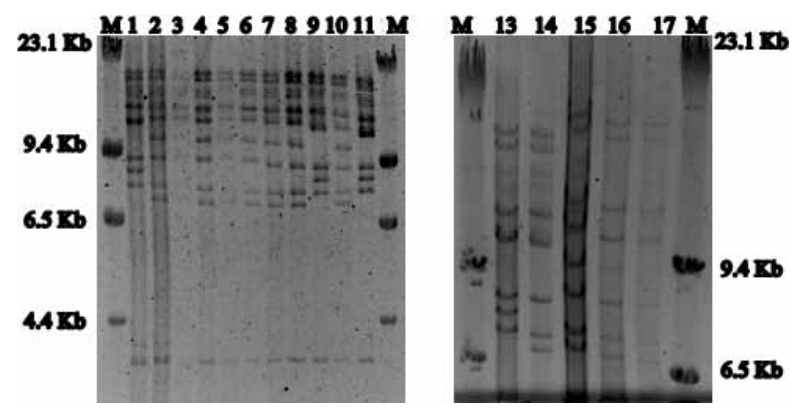

Figura 1. Patrón de clivado enzimático a modo de ejemplo de algunos de los aislamientos de campo de BoHV-1. M: Marcador de peso molecular (lambda HindIII, Invitrogen); Líneas 1 y 13: LA (BoHV-1.1 prototipo); Línea 2: K22 (BoHV-1.2b prototipo); Línea 14: ST (BoHV-1.2a prototipo); Líneas 9 y 11: aislamientos de campo subtipo 1.1; Líneas 3, 4, 5, 6 7, 8, 10, 15 y 16 aislamientos de campo con perfiles $1.2 \mathrm{~b}$; Línea 17: perfil del aislamiento 39 subtipos BoHV-1.2a. de las poblaciones de rebaños bovinos en Colombia tienen anticuerpos neutralizantes contra la infección por BoHV-1 ${ }^{10}$

En Brasil el porcentaje de seroprevalencia se ubica entre 19 y $85 \%{ }^{4}$, mientras que en Argentina el promedio es de $60 \%{ }^{8}$. Sin embargo, se dispone de información limitada sobre el tipo/subtipo de cepas circulantes en nuestro país. Para tener una vacuna eficiente es necesario y relevante saber qué subtipos de este virus están circulando actualmente.

En Argentina, un grupo de investigadores 9 caracterizaron por perfiles de restricción cuatro aislados de campo, tres de los cuales fueron BoHV-1.1 y el restante BoHV-1.2. Sin embargo, en este momento es necesario actualizar y aumentar el número de muestras/aislamientos analizados para determinar la situación de nuestro país en cuanto a los subtipos circulantes, su distribución en el tiempo, co-circulación y prevalencia de cada subtipo.

De acuerdo con las muestras analizadas en este trabajo el subtipo más prevalente es 1.2b (35 aislamientos de 44). Se cree que la transmisión de BoHV-1 por vía genital precede a la transmisión respiratoria que ocurrió aparentemente con el advenimiento de los feedlots ${ }^{12}$. Basados en tal observación y según los resultados aquí obtenidos, podríamos inferir que la prevalencia del subtipo $1.2 \mathrm{~b}$ podría deberse a la utilización más frecuente de sistemas extensivos de producción en Argentina.

Según los aislamientos aquí caracterizados, resulta interesante observar la co-circulación de los subtipos BoHV-1.1 y $1.2 b$ durante 30 años. Los aislamientos de BoHV-1.2a reportados en este trabajo fueron obtenidos de bovinos asintomáticos, uno de ellos aislado de semen durante el diagnóstico de rutina y el otro durante un muestreo aleatorio en un campo de producción mixta bovinos-búfalos.

En los últimos años la principal región pecuaria del centro de Argentina se ha dedicado a la producción de soja. Ello dio lugar a cambios importantes en la distribución del ganado bovino, como la intensificación de la carga de ganado en campos de menor calidad agrícola y la aparición de sistemas de producción mixtos (búfalobovino) ${ }^{5}$. Desde el punto de vista epidemiológico es interesante conocer que los alpha herpesvirus circulan en estos rebaños mixtos, siendo nuestro país endémico para los tipos BoHV-1, BoHV-5 ${ }^{9}$.

En búsqueda de una mejor comprensión de la epidemiología del herpesvirus bovino en el país, 44 aislamientos de campos de diferentes regiones agrícolas fueron examinados, pudiendo observarse que, independientemente del subtipo, las cepas mostraron tropismo diferente por las distintas mucosas. Del mismo modo, artículos brasileños han reportado casos en los cuales el cuadro clínico no correlacionó con el subtipo ${ }^{13}$.

De acuerdo con lo observado en este trabajo, en Argentina parecen circular todos los subtipos de herpesvirus bovino 1 descritos en la literatura (BoHV-1.1, BoHV-1.2a y b). Aunque se determinó que dos subtipos (BoHV-1.1 y BoHV-1.2b) circularon en el período 1982- 
2011, la mayoría de los aislamientos actuales son BoHV1.2b. Según la búsqueda efectuada, este sería el primer informe sobre la circulación del subtipo de BoHV-1 "1.2a" en Argentina, mostrando la circulación de tres subtipos de BoHV-1 como en otras partes del mundo.

De acuerdo al número de muestras analizadas, la mayoría de los aislamientos de campo, incluidos los actuales, pertenecen al subtipo " $1.2 \mathrm{~b}$ ". El subtipo "1.2a" sólo se aisló para este trabajo entre los años 2012-2015 y aparentemente es el subtipo menos prevalente en nuestro país.

La estrategia para controlar el BoHV-1, se basa en el diagnóstico y la vacunación. Las vacunas utilizadas en Argentina son inactivadas y generalmente se basan en el subtipo BoHV-1.1 (cepas de referencia de Los Angeles), ocasionalmente con la adición del virus BoHV5. El éxito de la vacunación eficaz contra el BoHV-1 debe tener en cuenta tanto la diversidad genética como antigénica del virus. Por esta razón, la caracterización de aislamientos recientes de BoHV-1 es necesaria para monitorear la aparición de variantes de importancia epidemiológica.

Dado que los subtipos de BoHV-1 están altamente relacionados antigénicamente, es probable que se produzca la protección cruzada de la vacuna actualmente utilizada en Argentina (subtipo 1.1). Sin embargo, hasta ahora no se han realizado ensayos experimentales en bovinos demostrando que una vacuna monovalente incluyendo solo el subtipo 1.1 proteja contra los otros subtipos de este virus.

Agradecimientos. Los autores desean agradecer a la Dra Mercedes Odeón por participar en la redacción del manuscrito y a los Dres Gastón Caspe y Daniel Benítez por colaborar en la recolección de muestras.

\section{REFERENCIAS}

1. Christensen L, Madsen K, Nylin B, Rønsholt L. 1996. A contribution to the systematization of bovine herpesvirus 1 based on genomic mapping by restriction fragment pattern analysis. Virus Res 46,: 177-182.
2. Claus M, Alfieri A, Folgueras A, Wosiacki S, Medici K, Alfieri A. 2005. Rapid detection and differentiation of bovine herpesvirus 1 and 5-glycoprotein $\mathrm{C}$ gene in clinical specimens by multiplex-PCR. J Virol Methods 128: 183188.

3. Edwards S, White H, Nixon P. 1990. A study of the predominant genotypes of bovid herpesvirus 1 found in the U.K. Vet Microbiol 22; 213-223.

4. Holz C et al. 2009. Soroprevalência de herpesvírus bovinos tipos 1 e/ou 5 no Estado do Rio Grande do Sul. Pesq Vet Bras 29: 767-773.

5. Konrad J et al. 2012. Experimental inoculation of Neospora caninum in pregnant water buffalo. Vet Parasitol 187: 72-78.

6. Miller J, Whetstone C, Maaten M. 1991. Abortifacient property of bovine herpesvirus type 1 isolates that represent three subtypes determined by restriction endonuclease analysis of viral DNA. Am J Vet Res 52, 458-461.

7. Muylkens B, Thiry J, Kirten P, Schynts F, Thiry E. 2007. Bovine herpesvirus 1 infection and infectious bovine rhinotracheitis. Vet Res 38: 181-209.

8. Odeón A et al. 2001. Seroprevalencia de la diarrea viral bovina, herpesvirus bovino y virus sincicial respiratorio en Argentina. Prev Vet Med 82: 216-220.

9. Pidone C, Galosi C, Echeverria M, Nosetto E, Etcheverrigaray M. 1999. Restriction endonuclease analysis of BHV-1 and BHV-5 strains isolated in Argentina. Zentralblatt für Veterinärmedizin 46: 453-456.

10. Ruiz J, Jaime J, Ramirez G, Vera V. 2012. Molecular and in vitro characterization of field isolates of bovine herpesvirus-1. Virol Sin 27: 26-37.

11. Schudel A, Carrillo B, Wyler R. 1986. Infections of calves with antigenic variants of bovine herpesvirus 1 (BHV-1) and neurological disease. Zentralbl Vet 33: 303310.

12. Thiry J, Dams L, Muylkens B, Thiry E. 2011. Isolation of cervid herpesvirus 1 from the genital tract of a farmed red deer in Northern France. Vet $J$ 187: 282-283.

13. Traesel C, Silva M, Spilki F, Weiblen R, Flores E. 2013. Nucleotide sequencing and phylogenetic analysis of the $3^{\prime}$ region of glycoprotein $\mathrm{C}$ gene of South American bovine herpesviruses 1 and 5. Res Vet Sci 94: 178-185. 